\title{
Simplified susceptibility analysis of shielded multiconductor transmission lines
}

\author{
B. Schetelig ${ }^{1}$, S. Parr ${ }^{1}$, R. Rambousky ${ }^{2}$, and S. Dickmann ${ }^{1}$ \\ ${ }^{1}$ Faculty of Electrical Engineering, Helmut-Schmidt-University/University of the Federal Armed Forces Hamburg, Germany \\ ${ }^{2}$ Bundeswehr Research Institute for Protective Technologies and NBC Protection (WIS) Munster, Germany
}

Correspondence to: B. Schetelig (schetelig@hsu-hh.de)

\begin{abstract}
In this contribution, a new kind of coupling parameters for shielded multiconductor transmission lines is presented. These parameters are easy to calculate and simplify the vulnerability analysis of shielded cables with multiple inner conductors. In this paper, the underlying theory is derived in detail. The gained insight is demonstrated with a sample measurement.
\end{abstract}

\section{Introduction}

If the susceptibility of complex structures in large volumes is analyzed, the attention has to be drawn to a smart integration of the transmission lines into the entire model of the structure. A numerical field analysis can be accelerated by separating the analysis into two parts. The field analysis is carried out numerically and afterwards, the cables are added using multiconductor transmission line theory. This approach is particularly favourable for shielded transmission lines.

Cable shields can be integrated into transmission line theory by introducing the so-called transfer impedance and transfer admittance. These quantities are well-defined for single conductor transmission lines. Transfer parameters for multiconductor transmission lines are determined in several ways in literature (e.g. Weitze, 1991; Kley, 1991; Degauque and Hamelin, 1993; Kasdepke, 1997). In this paper, a new definition of multiconductor coupling parameters is introduced that uses some approximations to simplify the description of the coupling process.

\section{Theory of the transfer parameters}

In this section, the theory of the transfer parameters is briefly reviewed to the extent as it is applied in the proposed procedure.

To describe the voltages and currents on shielded cables, the well-known equivalent circuits from transmission line theory are used. Because of the shield, which separates the inner conductors from the outer world, one circuit is used for each of these areas (Fig. 1). The inner system consists of the inner conductor and the inside of the shield (reference conductor). The outer system is made up by the outside of the shield and the remaining structure around the transmission line. The shield is assumed to be a good one, which allows the application of the "good shielding approximation" and the concept of electromagnetic topology (Baum, 1980). This permits to consider the influence of external electromagnetic fields on the inner conductor primarily as an indirect coupling via the cable shield. In this concept, the field couples to the shield and the resulting voltages and currents of the shield then cause interferences inside.

The outer and the inner systems are linked via the transfer impedance and the transfer admittance. They can be defined, using a short section of a cable (Fig. 2), as (Vance, 1978):

$$
\begin{aligned}
& Z_{\mathrm{T}}^{\prime}=\frac{1}{I_{\mathrm{S}}} \cdot \frac{d U_{\mathrm{i}}}{d z} ; I_{\mathrm{i}}=0, \\
& Y^{\prime}{ }_{\mathrm{T}}=-\frac{1}{U_{\mathrm{S}}} \cdot \frac{d I_{\mathrm{i}}}{d z} ; U_{\mathrm{i}}=0 .
\end{aligned}
$$

These two parameters can be found in the definitions of the distributed sources of the inner system (Fig. 1):

$U_{\mathrm{S}, \mathrm{i}}^{\prime}=Z^{\prime}{ }_{\mathrm{T}} \cdot I_{\mathrm{S}}$, 

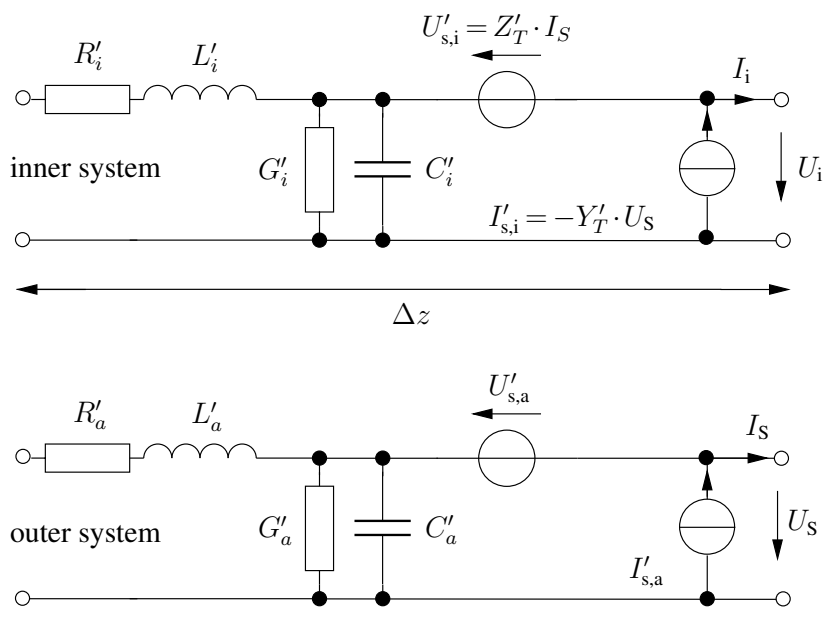

Fig. 1. Equivalent circuit of a shielded transmission line.

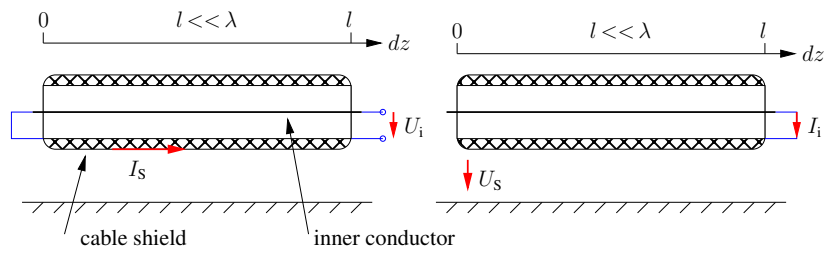

Fig. 2. Very small section of transmission line to define the transfer impedance (left) and transfer admittance (right).

$I^{\prime}{ }_{\mathrm{S}, \mathrm{i}}=-Y^{\prime}{ }_{\mathrm{T}} \cdot U_{\mathrm{S}} \cdot$

This description of cable shields can be expanded to multiconductor transmission lines. While the equivalent circuit of the outer system remains the same, the model of the inner system is replaced by the equivalent circuit of a multiconductor transmission line. In this model, each inner conductor is stimulated by individual distributed voltage and current sources. They can be described as follows:

$\boldsymbol{U}_{\mathrm{S}, \mathrm{i}}^{\prime}=\boldsymbol{Z}_{\mathrm{T}}^{\prime} \cdot I_{\mathrm{S}}$,

$\boldsymbol{I}_{\mathrm{S}, \mathrm{i}}^{\prime}=-\boldsymbol{Y}_{\mathrm{T}}^{\prime} \cdot U_{\mathrm{S}} \cdot$

These formulas use transfer parameter vectors of dimension $N$ to describe the coupling to a shielded $N$ conductor transmission line.

\section{Concept of simple equivalent coupling parameters for multiconductor transmission lines}

Previous work (Jung, 2003) analyzed industrial multiconductor cables with stranded wires. It was shown that the coupling parameters of the wires within such a cable usually are quite similar, if the cable is not mechanically damaged. This is valid for the absolute values and the phases. Other investigations show that even the difference between the transfer impedances of a conductor in the centre and a conductor at

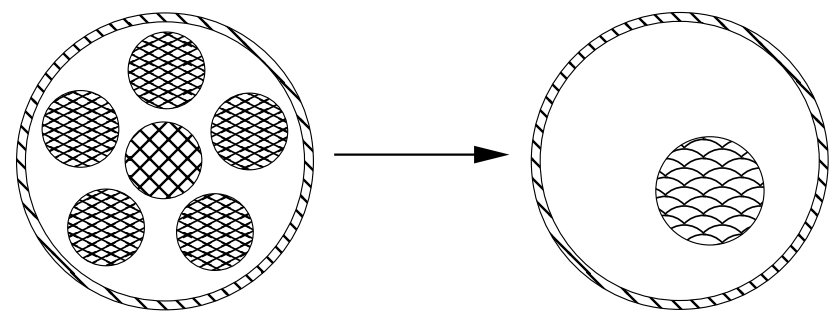

Fig. 3. Principle of using an equivalent cable description to define alternative transfer parameter.

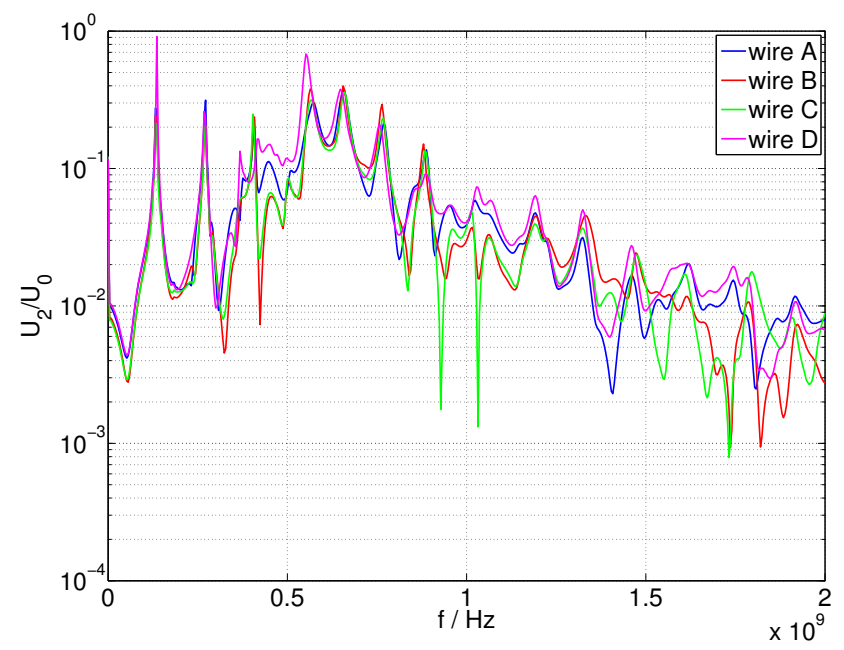

Fig. 4. Coupling to the four inner conductors of a shielded LiYCY transmission line.

the border of a bundle of conductors is rather small. The difference between the less important transfer admittances is slightly larger (Kasdepke, 1997).

These observations are of course only valid for cables without mechanical defects. If shielded multiconductor cables are bent by force, the coupling parameters of the individual wires can become different from each other. As it is usually not known throughout a susceptibility analysis, if there are mechanically stressed cables, these differences between the various wires cannot be taken into consideration, anyhow.

Therefore, in the following it is assumed that an external electromagnetic field causes quite similar interferences on the inner conductors. In Fig. 4, the shield of a four-wire-cable is excited at one end of the cable by a voltage $U_{0}$. The resulting voltages $U_{2, i}$ between each inner wire and the shield are measured at the other end (setup analogous to Fig. 5).

This property of approximately identical voltages and currents shall be used to simplify the calculation of the coupling parameters of multiconductor transmission lines. That for, the shielded multiconductor transmission line will be replaced by an equivalent model, which is made up by only one 


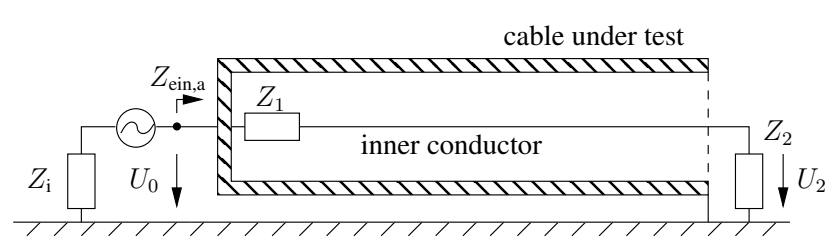

Fig. 5. Simplified setup to determine the transfer parameters of a shielded single conductor transmission line.

inner conductor and the shield (Fig. 3). Nevertheless, that does not mean that the inner conductors are simply shortcircuited or that all wires except one are neglected. Instead, the observation of almost identical currents and voltages from above is applied the following way:

Following (Andrieu, 2006), the differential equations of the inner multiconductor system,

$$
\begin{aligned}
-\frac{d}{d z}\left(\begin{array}{l}
U_{1} \\
U_{2} \\
\ldots \\
U_{N}
\end{array}\right) & =\left(\boldsymbol{R}^{\prime}+j \omega \boldsymbol{L}^{\prime}\right) \cdot\left(\begin{array}{l}
I_{1} \\
I_{2} \\
\ldots \\
I_{N}
\end{array}\right), \\
-\frac{d}{d z}\left(\begin{array}{l}
I_{1} \\
I_{2} \\
\ldots \\
I_{N}
\end{array}\right) & =\left(\boldsymbol{G}^{\prime}+j \omega \boldsymbol{C}^{\prime}\right) \cdot\left(\begin{array}{l}
U_{1} \\
U_{2} \\
\ldots \\
U_{N}
\end{array}\right),
\end{aligned}
$$

can be simplified. This is done by using the assumptions

$$
\begin{aligned}
& U_{\mathrm{eq}}=U_{1}=U_{2}=\ldots=U_{N}=\frac{\sum_{n=1}^{N} U_{i}}{N}, \\
& I_{\mathrm{eq}}=\sum_{n=1}^{N} I_{n}=N \cdot I_{1} .
\end{aligned}
$$

Then, the Eqs. (7) and (8) reduce from dimension $N$ to the dimension 1:

$$
\begin{aligned}
& -\frac{d}{d z} U_{\mathrm{eq}}=\left(R_{\text {eq }}^{\prime}+j \omega L_{\text {eq }}^{\prime}\right) \cdot I_{\text {eq }} \\
& -\frac{d}{d z} I_{\text {eq }}=\left(G_{\text {eq }}^{\prime}+j \omega C_{\text {eq }}^{\prime}\right) \cdot U_{\text {eq }} .
\end{aligned}
$$

$R_{\mathrm{eq}}^{\prime}, L_{\mathrm{eq}}{ }^{\prime}, G_{\mathrm{eq}}^{\prime}$ and $C_{\mathrm{eq}}{ }^{\prime}$ are the results of merging the rows and columns:

$$
\begin{aligned}
R^{\prime} \mathrm{eq} & =\frac{\sum_{i=1}^{N} \sum_{j=1}^{N} R^{\prime}{ }_{i j}}{N^{2}}, \\
L^{\prime} \mathrm{eq} & =\frac{\sum_{i=1}^{N} \sum_{j=1}^{N} L^{\prime}{ }_{i j}}{N^{2}}, \\
G^{\prime} \mathrm{eq} & =\sum_{i=1}^{N} \sum_{j=1}^{N} G^{\prime}{ }_{i j}, \\
C^{\prime} \mathrm{eq} & =\sum_{i=1}^{N} \sum_{j=1}^{N} C^{\prime}{ }_{j j},
\end{aligned}
$$

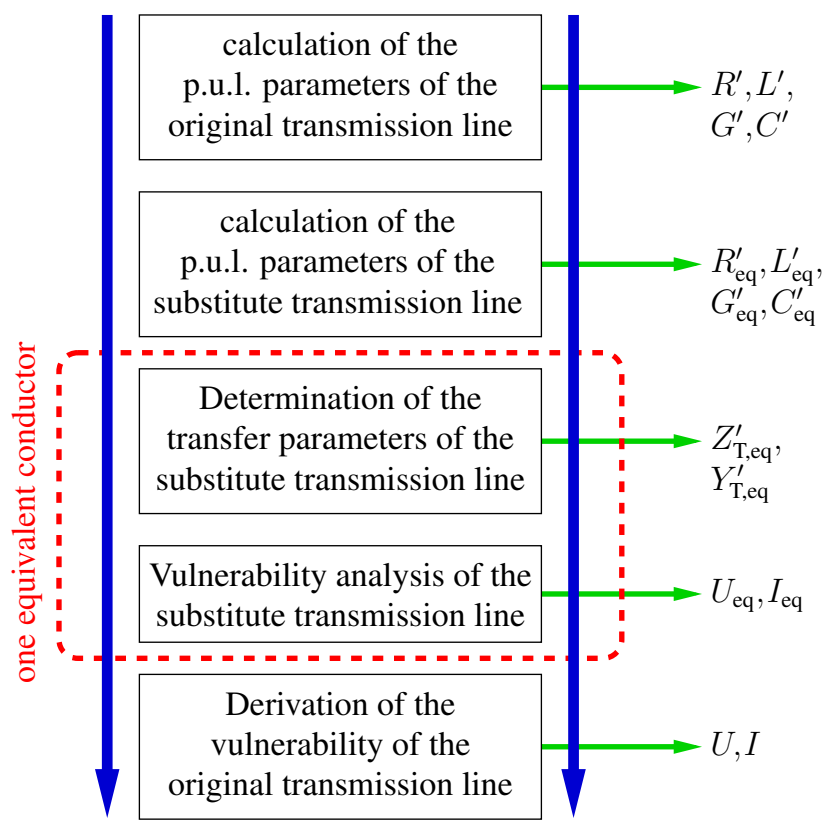

Fig. 6. Summary of the concept of alternative coupling parameters.

with $i j$ : indexation of the matrix elements of the prevailing original per unit length parameters.

Equations (11) and (12) describe a shielded equivalent transmission line with only one inner conductor. This substitute system is solely defined by its equivalent per unit length parameters. The corresponding and exact substitute geometry is neither known nor of interest for this application.

This substitute cable can now be used to calculate the equivalent transfer impedance and transfer admittance. They are defined as:

$$
\begin{aligned}
& Z_{\mathrm{T}, \mathrm{eq}}^{\prime}=\frac{U_{\mathrm{eq}}}{I_{\mathrm{S}}}, \\
& Y_{\mathrm{T}, \mathrm{eq}}^{\prime}=\frac{I_{\mathrm{eq}}}{U_{\mathrm{S}}} .
\end{aligned}
$$

This means that coupling to the inner system of a multiconductor transmission line can be described by scalar coupling parameters. The consequence is that one can now use the simple procedure for a coaxial line to describe the multiconductor coupling process.

\section{Measurement setup to determine the coupling parameters}

The application of the concept of equivalent coupling parameters is independent from the measurement procedure used to determine the parameters. A well-known approach is the "triaxial setup". This measurement setup can be simplified as shown in Fig. 5. The measurement principle is, to excite the shield via the source $U_{0}$ and to measure the 


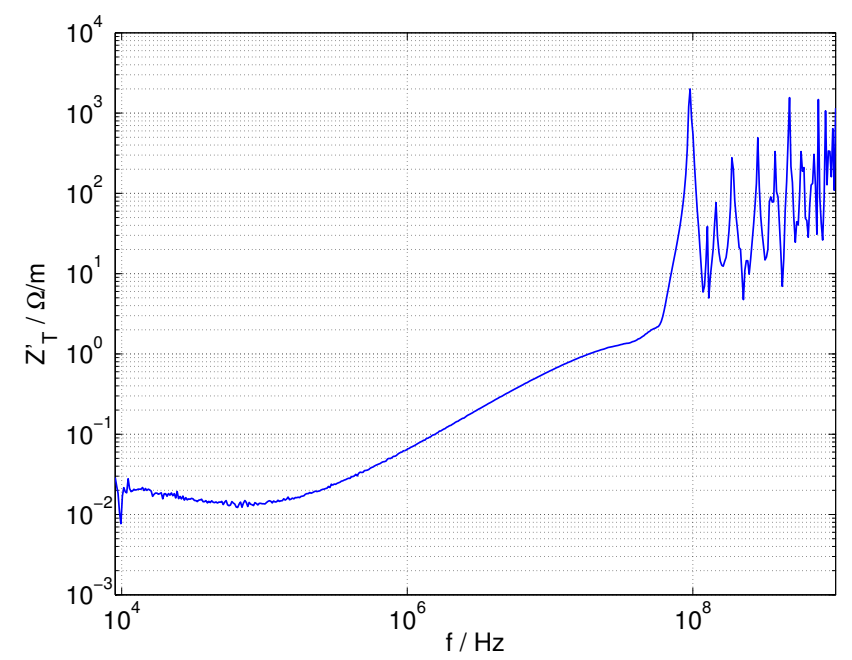

Fig. 7. Absolute value of the equivalent transfer impedance for a LiYCY multiconductor transmission line (4 inner wires).

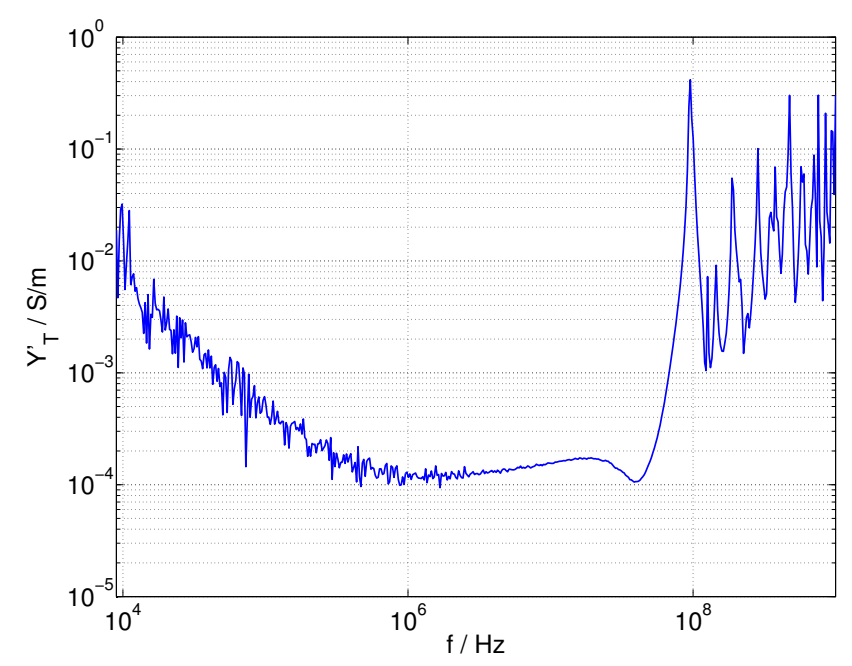

Fig. 8. Absolute value of the equivalent transfer admittance for a LiYCY multiconductor transmission line (4 inner wires).

voltage $U_{2}$ between the inner conductor and the shield. Using transmission line theory, these quantities can be used to calculate the transfer impedance and the transfer admittance (Tiedemann, 2001).

There is no difference between the calculation of the transfer parameters of a physical coaxial line and of the substitute transmission line from above. The equivalent cable is described in the usual notation of a single conductor with the per unit length parameters $R_{\text {eq }}^{\prime}, L_{\text {eq }}^{\prime}, G_{\text {eq }}^{\prime}, C^{\prime}$ eq and the equivalent voltage $U_{\text {eq. }}$. If these equivalent substitute quantities are used instead of physical ones, any measurement setup can be used to determine the equivalent coupling parameters of Eqs. (17) and (18).

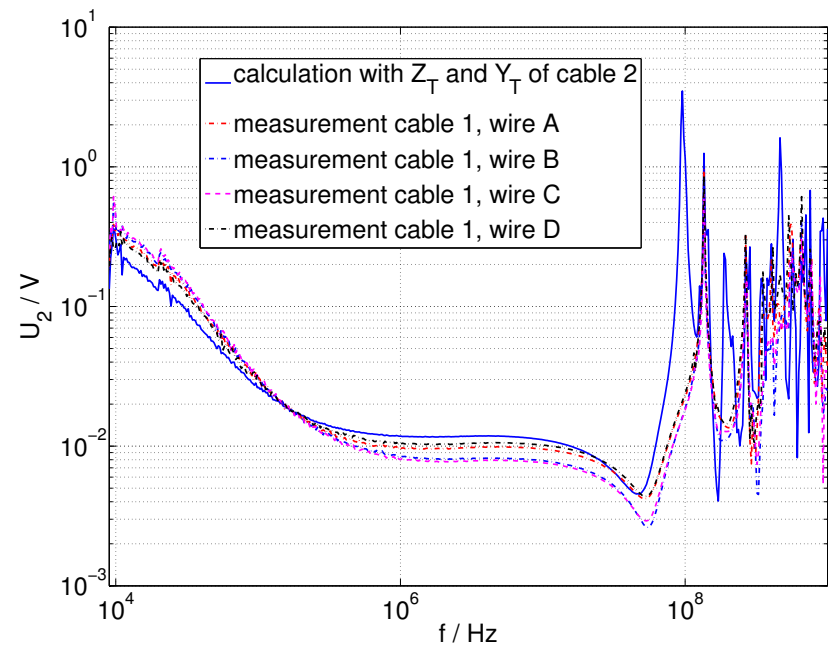

Fig. 9. Calculation of the induced voltage at an inner conductor of a shielded LiYCY cable.

\section{Susceptibility analysis with equivalent coupling parameters}

The overview of the procedure (Fig. 6) shows that the first three steps are completed so far. The fourth step is to do the susceptibility analysis of the structure. This includes calculating the distributed sources of the inner system using the quantities $Z^{\prime}$ T,eq and $Y^{\prime}$ T,eq. Common single conductor transmission line theory can then be used to calculate the interferences $U_{\mathrm{eq}}, I_{\mathrm{eq}}$ at the end of the lines (Tesche et al., 1997). After that, Eqs. (9) and (10) can be used to derive the interferences $U, I$, of the original transmission line.

\section{Conversion of the equivalent coupling parameters to the conventional ones}

The equivalent coupling parameters in combination with the equivalent single conductor notation of the inner system can be used to determine easily the interferences on the inner conductors. Nevertheless, it is possible to transform the equivalent transfer parameters to the conventional description. This might be useful, if there is already a multi conductor transmission line implementation for the inner system.

The relationship can be drawn from the definitions of the parameters: Using the assumption of approximately identical interferences on the inner conductors, the conventional transfer impedance can be written as:

$\boldsymbol{Z}_{\mathrm{T}}^{\prime}=\left(\begin{array}{c}\frac{U_{1}}{{ }_{\mathrm{S}}} \\ \ldots \\ \frac{U_{N}}{I_{\mathrm{S}}}\end{array}\right)=\frac{U_{1}}{I_{\mathrm{S}}} \cdot \mathbf{1}^{N \times 1}$. 
Using the definition of the equivalent transfer impedance $Z_{\text {T,eq }}^{\prime}$ (Eqs. 17 and 9), the demanded link can be established:

$Z^{\prime}{ }_{\mathrm{T}}=Z^{\prime}{ }_{\mathrm{T}, \mathrm{eq}} \cdot \mathbf{1}^{N \times 1}$.

Under the same conditions, the conventional transfer admittance is:

$\boldsymbol{Y}_{\mathrm{T}}^{\prime}=\left(\begin{array}{c}\frac{I_{1}}{U_{\mathrm{S}}} \\ \ldots \\ \frac{I_{N}}{U_{\mathrm{S}}}\end{array}\right)=\frac{I_{1}}{U_{\mathrm{S}}} \cdot \mathbf{1}^{N \times 1}$.

For the equivalent transfer admittance, the following is valid:

$Y_{\mathrm{T}, \mathrm{eq}}^{\prime}=\frac{I_{\mathrm{eq}}}{U_{\mathrm{S}}}=\frac{\sum_{n=1}^{N} I_{n}}{U_{\mathrm{S}}}=N \cdot \frac{I_{1}}{U_{\mathrm{S}}}$.

The second demanded link then becomes:

$\boldsymbol{Y}_{\mathrm{T}}^{\prime}=\frac{Y^{\prime} \mathrm{T}, \mathrm{eq}}{N} \cdot \mathbf{1}^{N \times 1}$.

\section{Sample measurements}

The procedure which was presented before was applied on a shielded LiYCY cable with four inner conductors. The structure was condensed to a shielded single conductor line. The equivalent per unit length parameters and the induced voltage $U_{\text {eq }}$ were derived. This data then is used to calculate the scalars equivalent transfer impedance (Fig. 7) and transfer admittance (Fig. 8).

If these coupling parameters are applied to the susceptibility analysis of the cable under test, the induced voltage at the inner conductor of the equivalent cable can be calculated (Fig. 9). The transfer parameters are calculated with the measured data of the "cable piece no. 2". This data then is applied on a susceptibility analysis of the "cable piece no. 1".

Because of Eq. (9), the values of the induced voltages of the original cable are the same as the induced voltage of the substitute. That means, no further calculation has to be carried out.

\section{Conclusions}

The procedure, which is presented here, is a considerable simplification when calculating the coupling parameters of industrial multiconductor transmission lines with stranded wires. The deviations are quite acceptable. The application to the susceptibility analysis of the cables under test results in a reduced complexity, since only a simple single conductor transmission line has to be considered. The total procedure is summarized in Fig. 6.

\section{References}

Andrieu, G.: Elaboration et application d'une méthode de faisceau équivalent pour l'étude des couplages électromagnetiques sur réseaux de câblages automobiles, Ph.D. thesis, Université de Lille, 2006.

Baum, C. E.: Electromagnetic topology: A formal approach to the analysis and design of complex electronic systems, Tech. Rep., Interaction Notes - Note 400, available at: http://www.ece.unm. edu/summa/notes/In/0400.pdf, 1980.

Degauque, P. and Hamelin, J.: Electromagnetic Compatibility, Oxford Science Publications, 1993.

Jung, L.: Einfluß von Schirminhomogenitäten bei Mehrleiterkabeln auf die komplexe Transferimpedanz, Ph.D. thesis, Technische Universität Hamburg-Harburg, 2003.

Kasdepke, T.: Simulation von Störströmen auf geschirmten mehradrigen Kabeln, Ph.D. thesis, Technische Universität Hamburg-Harburg, 1997.

Kley, T.: Optimierte Kabelschirme Theorie und Messung, Ph.D. thesis, Eidgenössische Technische Hochschule Zürich, 1991.

Tesche, F. M., Ianoz, M. V., and Karlsson, T.: EMC Analysis Methods and Computational Methods, Wiley, 1997.

Tiedemann, R.: Schirmwirkung koaxialer Geflechtsstrukturen, Ph.D. thesis, Technische Universität Dresden, 2001.

Vance, E. F.: Coupling to shielded cables, John Wiley \& Sons, Inc., 1978.

Weitze, F.: Ausbreitung eingekoppelter Störströme auf abgeschirmte Leitungen, Ph.D. thesis, Technische Universität Hamburg-Harburg, 1991. 